\title{
Turbulent kinetic energy budget in a wall-mounted cylinder wake using PIV measurements
}

\author{
Ikhennicheu Maria ${ }^{1,2}$, Druault Philippe ${ }^{3}$, Gaurier Benoit ${ }^{1}$, Germain Gregory ${ }^{1}$
}

1 IFREMER, Marine Structure Laboratory, 150 Quai Gambetta 62200 Boulogne-sur-Mer, France

2 INNOSEA, 1 rue de la Nöe, CS 1210244321 Nantes CEDEX 03, France

${ }^{3}$ Sorbonne Université, CNRS, UMR 7190, Institut Jean Le Rond d'Alembert, F-75005 Paris, France

Email addresses : maria.ikhennicheu@innosea.fr ; philippe.druault@sorbonne-universite.fr ; gregory.germain@ifremer.fr

\begin{abstract}
:
In high flow velocity areas, turbulence intensity is high and flow variations may have a major impact on tidal turbines behaviour. Previous studies show that a square wall-mounted cylinder produces a very extended and energetic wake. In this work, two-component PIV measurements are conducted in the symmetry plane of the flow in order to evaluate the Turbulent Kinetic Energy (TKE) budget. This analysis enables to show how the TKE, produced in the shear-layer region, is re-distributed and dissipated within the flow. The Large-Eddy PIV method enables to obtain full spatial maps of dissipation and to assess the validity of the constant involved in the spectral method. Results show that the production occurs when the Reynolds shear stress is the more intense, so is the dissipation. Energy is then transported through turbulent motion into the outer flow and swept to the bottom due to the pressure gradient effects. Production is directly due to the periodic vortex shedding unlike for other terms. A better description of the energy exchanges in the turbulent wake flow is obtained. This knowledge is important for the performance determination of a tidal turbine positioned in such an obstacle wake which affects the flowinduced dynamic load on turbine.
\end{abstract}

\section{Highlights}

- Tests are carried out on a wall-mounted cylinder representative of seabed elements, in Froude similitude with high Reynolds number. PIV measurements are performed in vertical measurement planes and spatial analyses are performed. The turbulent kinetic energy budget is calculated in the cylinder wake.

Keywords : Turbulence, Experimental trials, Wall-mounted cylinder, PIV, LDV, Kinetic energy budget 


\section{Introduction}

Industrial projects have started to emerge in tidal energy harvesting. There is a strong tidal potential in Europe and over 25\% of the European potential is located in French waters [EDF (2010)]. In-situ, strong velocity variations exist [Myers and Bahaj (2005)]. The flow perturbations can come from waves, inflow turbulence, coast or bathymetry effects [Ouro and Stoesser (2018)]. Such flow variations are causing a high turbulence rate in the water column. Moreover, due to bathymetry variations, some turbulent events can sometimes rise up to the surface and erupt to create a boil as it is observed in the Alderney race [Myers and Bahaj (2005)].

[Ikhennicheu et al. (2019b)] presented a case study comparison of various elementary obstacles used to reproduce at scale bathymetry variations. They concluded that a surface mounted cylinder produces the most energetic wake that extends up to the free surface. In this case, large scale turbulent structures are periodically emitted from its wake and their diameter is similar to the one of a turbine at the same scale [Ikhennicheu et al. (2019c)]. These high energetic flow structures induce some mean velocity shear and thus a turbulent kinetic energy (TKE) production term. This work proposes a better understanding of the transportation of the turbulent kinetic energy in the wake of two-dimensional obstacle related to realistic bathymetry variations. The knowledge of the inflow turbulence that will potentially impact a turbine is of great interest for optimizing the turbine performance [Duran Medina et al. (2017)]. Indeed, the turbulent inflow condition plays a crucial role not only on both fatigue and mean loads acting on hydrodynamics turbine [Mycek et al. (2014)] but also on the wake evolution behind the turbine. For the development of tidal turbine farms, a better knowledge of the turbine wake under specific incoming turbulent flow conditions is essential to optimize the turbines location. In order to know if such inflow can be critical for tidal turbines behaviour, specific studies were initiated [Ikhennicheu et al. (2019a)]. But today, a better knowledge of the flow characteristics in the cylinder wake is still needed, particularly from an energetic point of view. Such description will allow a more thorough validation of numerical models [Mercier et al. (2020)] which will then provide precise flow data in tidal sites. This work highlights how the Large-Eddy PIV approach enables us to obtain full spatial maps of dissipation and also to compare it with the spectral method and assess of the validity of the constant used for the dissipation rate estimation. The description of the energy exchange in the turbulent wake flow will permit to determine the performance of a tidal turbine positioned in such an obstacle wake. 


\begin{tabular}{|c|c|c|c|}
\hline Reference & Obstacle - Flow & Data & Re $\left(10^{3}\right)$ \\
\hline \hline Acharya et al. (1994) & 2D rib & k- $\epsilon$ / LDV & 240 \\
Hussein et al. (1996) & Cube & LDA & 80 \\
Castro et al. (2006) & TBL rough wall & HWA / LDA & $12^{*}$ \\
Panigrahi et al. (2008) & Permeable ribs & PIV & 6 \\
Shah and Tachie (2008) & Square rib & PIV & 3 \\
Panigrahi (2009) & Detached cylinder & PIV & 11 \\
Dey et al. (2011) & Sphere & ADV & 16 \\
Cheng et al. (2013) & 2D block & LES & 50 \\
Ricardo et al. (2014) & Array cylinders & LDA / PIV & 1 \\
Blackman et al. (2017) & TBL rough wall & PIV & 190 \\
\hline Present work & Square cylinder & PIV / LDV & 250 \\
\hline
\end{tabular}

Table 1: Previous experimentally and numerically TKE budget investigations determined in similar wall mounted obstacle flow configurations. Reynolds number is based on the height of the obstacle (apart $R e^{*}$ which is based on boundary layer momentum thickness). LES: Large Eddy Simulation. HWA: Hot-Wire Anemometry. ADV: Acoustic Doppler Velocimetry. LDV: Laser Doppler Velocimetry. LDA: Laser Doppler Anemometry. PIV: Particle Image Velocimetry.

Many studies have focused on turbulent flow over and behind a surfacemounted obstacle due to its important implication in many engineering applications. However, only a few studies detailed the TKE budget obtained in a flow configuration close to the current one. These studies are given in table 1 where the nature of available data (experimental or numerical) and the Reynolds number of the flow configuration are mentioned.

In this study, we propose to analyze previous LDV and PIV measurements [Ikhennicheu et al. (2019a)] obtained in the wake of a horizontal wallmounted square cylinder. This flow configuration and measurement methods have specific characteristics which greatly differ from previous investigations (see table 1). First, the Reynolds number based on the mean upstream flow velocity and the cylinder height is equal to $2.5 \times 10^{5}$. Second, to fully investigate the near and far wake of the cylinder, large PIV measurement planes $\left(0.5 \mathrm{~m}^{2}\right)$ with a high spatial discretization $(1.3 \mathrm{~cm}$, in each direction) have been used. The purpose is then to determine each term of the equation of conservation of the turbulent kinetic energy. Hence, this works proposed to perform a TKE budget based on PIV measurements at Reynolds number much higher than existing works (10 to 100 times higher), thus providing data closer to in situ conditions. 
The determination of the turbulent dissipation rate $\epsilon$ appearing in the TKE budget requires turbulent velocity gradients which can not be entirely and accurately computed from 2D-PIV. Many studies focused on the estimation of the dissipation rate from PIV measurements [Saarenrinne and Piirto (2000), Zaripov et al. (2019)]. The remaining velocity gradients are generally estimated under isotropy assumption or other hypothesis. But the PIV resolution especially in our case, is too large to access the dissipative smallest scales, smaller than the Kolmogorov scale. To address this problem, several methods have been developed [Sheng et al. (2000)], such as the Large-Eddy PIV method and the spectral method. The former follows the same concept of Large Eddy simulation where the large-scale flow structures are computed while the smallest ones are modelled thanks to a sub-grid scale model. This assumes that the turbulent kinetic production is only due to large scale structures which is true for high Reynolds number turbulent flow and the turbulent energy dissipation comes from the smallest scale structures. The energy transfer takes place in the inertial area determined by the properties of the flow. This method relies then on the determination of a spatial filter size applied to PIV measures for the separation of involved flow scales. To access the spatial scale filtering process, the direct energy transfer method presented in [Natrajan and Christensen (2006)] can be considered. It allows a direct calculation of the energy transfer between the large-scale filtered turbulence and the small-scale structures. Conversely, the spectral method is based on the local isotropy of the flow and the frozen turbulence Taylor's assumption. These two methods will be successively implemented in this work to access the energy dissipation rate, justifying the use of LDV measurements in present investigation for the spectral method application.

The main objective is to characterize the spatial distribution of each term (the production, the turbulent transport, the pressure transport, the convection and the viscous dissipation) in the near- and far-wake of the surface mounted $2 \mathrm{D}$ cylinder. A better description of the energy exchanges in the turbulent wake flow is important for the determination of the performance of a tidal turbine positioned in such an obstacle wake which affects the flow-induced dynamic load on turbine. It is also of great importance for the analysis of the accuracy of the closure models used in the numerical simulation.

In this paper, the experimental set-up is first described with a presentation of the tank and the used velocimetry techniques. Then, the average flow past the wall-mounted cylinder is briefly reminded. Finally, the decomposition of the turbulent kinetic energy budget is proposed and results for the wall-mounted cylinder cases are presented. 


\section{Experimental set-up}

The tests are carried out in the wave and current circulating flume tank of IfREMER located in Boulogne-sur-Mer (France) presented in figure 1(a). The test section is: $18 m$ long $\times 4 m$ wide $\times 2 m$ high. In this work, the three instantaneous velocity components are denoted $(U, V, W)$ along the $(X, Y, Z)$ directions respectively (figure 1(b)). Each instantaneous velocity component is separated into a mean value and a fluctuating part according to the Reynolds decomposition: $U=\bar{U}+u^{\prime}$, where an overbar indicates the time average. The incoming flow $\left(U_{\infty}, V_{\infty}, W_{\infty}\right)$ is assumed to be steady and constant. By means of a grid and a honeycomb (that acts as a flow straightener) placed at the inlet of the working section (see figure 1(b)), a turbulent intensity of $I=1.5 \%$ is achieved for a flow velocity of $1 \mathrm{~m} / \mathrm{s}$. Turbulence intensity $I$ in the incoming flow is defined as follows, where $\sigma$ is the standard deviation:

$$
I=100 \sqrt{\frac{\frac{1}{3}\left(\sigma(U)^{2}+\sigma(V)^{2}+\sigma(W)^{2}\right)}{{\overline{U_{\infty}}}^{2}+{\overline{V_{\infty}}}^{2}+\bar{W}_{\infty}^{2}}}
$$

Table 2: in-situ and experimental conditions (1:20 scale).

\begin{tabular}{|c|c|c|c|c|c|c|}
\hline & Scale & $\begin{array}{c}U_{\infty} \\
{[\mathrm{m} / \mathrm{s}]}\end{array}$ & $\begin{array}{c}\text { Rugosity } \\
\mathrm{H}[\mathrm{m}]\end{array}$ & $\begin{array}{c}\text { Depth D } \\
{[\mathrm{m}]}\end{array}$ & $\begin{array}{c}R_{e}= \\
\frac{H U_{\infty}}{\nu}\end{array}$ & $\begin{array}{c}F_{r}= \\
\frac{U_{\infty}}{\sqrt{g D}}\end{array}$ \\
\hline Alderney Race & 1 & 5 & 5 & 40 & $2.5 \times 10^{7}$ & 0,25 \\
\hline Flume tank & $1 / 20$ & 1 & 0.25 & 2 & $2.5 \times 10^{5}$ & 0.23 \\
\hline
\end{tabular}

The wall-mounted cylinder case is chosen to be representative of real-life conditions (Alderney race, [Ikhennicheu et al. (2019a)]). As there is no obstacle upstream of the cylinder: the upstream flow is a simple boundary layer developing over the tank floor. Hence the experimental set-up represents a bump considerably higher than its neighbours. To consider turbulent events interaction with the free surface, experiments shall be achieved in Froude similitude (see table 2) where $F_{r}=\frac{U_{\infty}}{\sqrt{g D}}$, with $g$ the gravity and $D$ the tank depth. Furthermore, Reynolds number must be as high as achievable to be closer to real conditions. The obstacle of interest in this study is a wallmounted cylinder of aspect ratio $A_{R}=$ Width/Height $=6$. This obstacle represents a key bathymetric element in the area of interest at a 1:20 scale (see table 2). In the following, non dimensional lengths are used for all parameters indexed by ${ }^{*}: x^{*}=x / H, y^{*}=y / H, z^{*}=z / H$ and $\delta^{*}=\delta / H$, with $\mathrm{H}$ the cylinder height $(H=0.25 \mathrm{~m})$. At the obstacle position, the boundary 
layer height $\delta^{*}$ equal to 1.3 is calculated as $\delta_{95}^{*}=z^{*}\left(\bar{U}=0.95 \times \overline{U_{\infty}}\right)$.

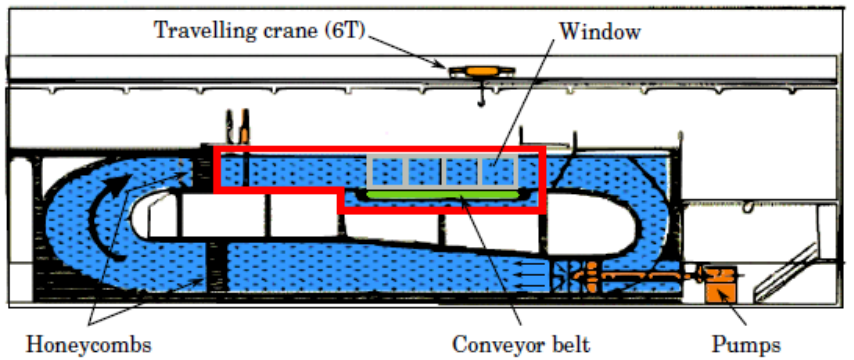

(a)

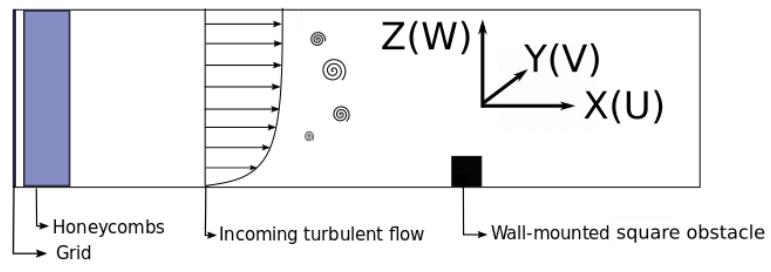

(b)

Figure 1: IfREMER Flume tank description and schematic view of the experimental set-up.

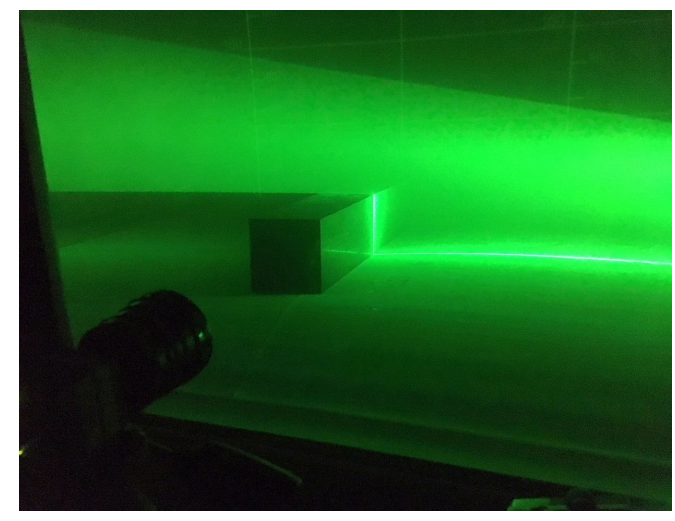

Figure 2: Cylinder during PIV measurements.

To characterize the flow, two Laser Velocimetry techniques are used: LDV (Laser Doppler Velocimetry) and PIV (Particle Image Velocimetry) (see figure 2). Beforehand, the tank is seeded with $10 \mu \mathrm{m}$ diameter silver coated glass micro-particles. For the PIV measurements, a Nd-YAG 
Laser GEMINI-Like is used: power is $200 \mathrm{~mJ}$ per probe and wavelength is $532 \mathrm{~mm}$. The laser is synchronized with a Camera FlowSEns EO-2M 1600pix $\times 1200$ pix that makes double images with a time step of $1600 \mu \mathrm{s}$. A particle is detected on 3 to 5 pixels. Cross-correlation peak intensity is between 0.3 and 0.8 and peak detectability (ratio of the tallest correlation peak and the second tallest correlation peak [Adrian and Westerweel (2011)]) is 8 in average. PIV acquisitions are made for $150 s$, hence 2250 double images are taken with a $15 H_{z}$ acquisition frequency. Planes dimensions are $1153 \times 430 \mathrm{~mm}^{2}$. The data are post processed with the software Dynamic Studio. The displacement of particles is calculated using a Cross-Correlation on 32 pix $\times 32$ pix interrogation windows with $50 \%$ overlap [Meinhart et al. (1993)]. Outliers are replaced with the Universal Outlier Detection [Westerweel and Scarano (2005)]. Precisions on the method can be found in [Ikhennicheu et al. (2019c)], the resolution being: $d_{x}=d_{z}=11.6 \mathrm{~mm}$.

The LDV measurements are made using a 2D DANTEC FiberFLOW system. The probe is positioned horizontally for $(U, V)$ measurements at various streamwise positions along the $\mathrm{Z}$ axis. With LDV measurements, the acquisition frequency is not constant. It depends on the number of particles passing through the measurement volume. Then, a re-sampling is done in the post processing. Based on previous works performed in the tank [Durán Medina et al. (2015)], the re-sampling is done using the mean sample rate of the measurement set considered. At a specific streamwise position, $f_{e}$ varies from 70 to $270 \mathrm{H}_{z}$ depending on the turbulent agitation in the water column. Various sources of experimental errors can be identified for both techniques, they are detailed in [Ikhennicheu et al. (2019c)].

As both measurement methods will be used to access the fluctuating velocity field allowing the computation of each term of the TKE energy budget, a first comparison of LDV and PIV results has been done. It was demonstrated that both LDV and PIV measured velocity fields are in good agreement (mean flow, Reynolds tensor, spectral content, as well as the convergence of the first and second order flow statistics) [Ikhennicheu (2019)].

The cylinder wake has been extensively described in [Ikhennicheu et al. (2019c)]. Maps of average streamwise velocity component, $\bar{U}$ and Reynolds shear stress, $\overline{u^{\prime} w^{\prime}}$ are represented in figure 3. In these figures, the classical behaviour of the flow past a wall-mounted element can be observed: the flow separates at the leading edges of the cylinder into the outer steady region and the recirculation area downstream of the cylinder. A shear layer develops in-between (see figure 3(b)) and then the flow reattaches. 


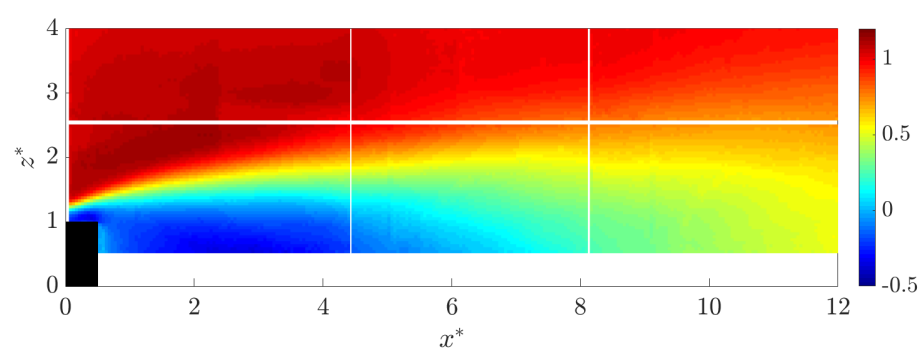

(a) $\bar{U} / U_{\infty}$

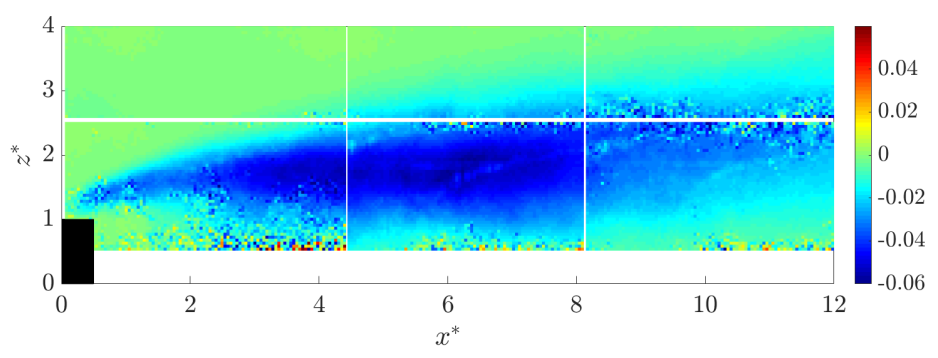

(b) $\overline{u^{\prime} w^{\prime}} / U_{\infty}^{2}$

Figure 3: Maps of average streamwise velocity component (top) and Reynolds shear stress $\overline{u^{\prime} w^{\prime}}$ (bottom), using PIV measurements.

[Ikhennicheu et al. (2019c)] explained that, in the symmetry plane, the wake extends towards the surface due to the negligible influence of side effects. The transverse component is not impacted by the cylinder wake (figure 4). At $x^{*}=5.5$ and $z^{*}=4$, the values are $U / U_{\infty}=0.64 \pm 0.2$, $V / U_{\infty}=0.00 \pm 0.04$ et $W / U_{\infty}=-0.05 \pm 0.16$. Hence, in the symmetry plane, the wake is developing in the $(x, z)$ plane and has a $2 \mathrm{D}$ aspect.

In the cylinder wake, large scale turbulent structures are generated and they rise up in the water column. Figure 4 (right hand side) displays the spectrum of the fluctuating $u$ component extracting at $\left(x^{*}, z^{*}\right)=(2,2)$. It shows that these structures are shed from the cylinder at a frequency of $0.3 \mathrm{~Hz}$. The physical description of this phenomenon, presented in [Ikhennicheu et al. (2019c)], allowed to validate some numerical developments performed to study high energetic sites, where currents reach $5 \mathrm{~m} / \mathrm{s}$ [Mercier (2019)]. This study showed that, even if the mean flow velocity comparisons are good, the turbulent characteristics especially the energy exchanges in the flow have to be better characterised not only for numerical model im- 

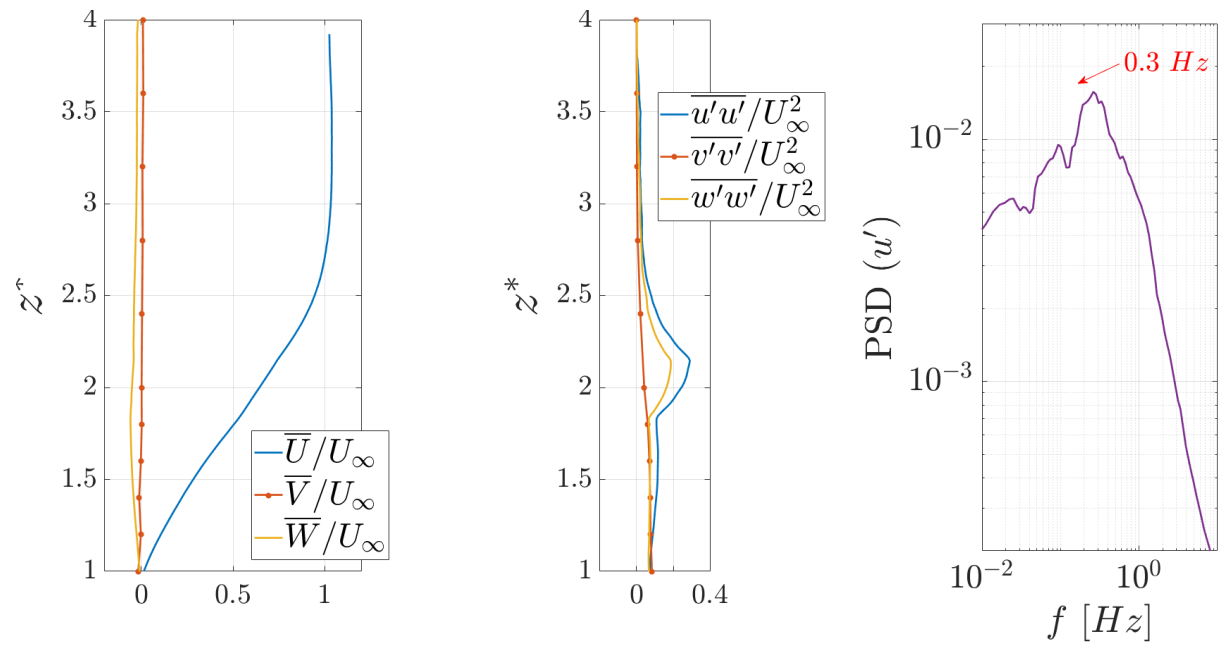

Figure 4: Left and center: Profiles of average and shear stress velocity components in $x^{*}=5.5$ and $y^{*}=0 . V$ is measured using LDV and $(U, W)$ using PIV. Right: $\operatorname{PSD}\left(u^{\prime}\right)$ measured with LDV at $x^{*}=2, z^{*}=2$.

provements but also for a better understanding of the inlet flow condition that could impact a turbine.

\section{Turbulent kinetic energy budget}

\subsection{Description of the method}

For an incompressible Newtonian fluid, the governing equations for the fluid movements are the equations of continuity and the Navier-Stokes equations. With $k=\frac{1}{2} \overline{u_{i}^{\prime} u_{i}^{\prime}}$ the turbulent kinetic energy, [Hinze (1975)] described the turbulent kinetic energy budget as follows:

$$
\begin{aligned}
\frac{\partial k}{\partial t}+\underbrace{\overline{U_{i} \frac{\partial k}{\partial x_{i}}}}_{C}= & -\underbrace{\frac{1}{\rho} \frac{\partial}{\partial x_{i}} \overline{u_{i}^{\prime} p^{\prime}}}_{\Phi}-\underbrace{\frac{1}{2} \frac{\partial}{\partial x_{j}} \overline{u_{i}^{\prime} u_{i}^{\prime} u_{j}^{\prime}}}_{T}-\underbrace{\overline{u_{i}^{\prime} u_{j}^{\prime}} \frac{\partial \overline{U_{i}}}{\partial x_{j}}}_{P}+ \\
& \underbrace{\nu \frac{\partial}{\partial x_{i}} \overline{\left(\frac{\partial u_{i}^{\prime} \partial u_{j}^{\prime}}{\partial x_{j} \partial x_{i}}\right)}}_{D_{\mu}}-\underbrace{\nu \overline{\left(\frac{\partial u_{i}^{\prime}}{\partial x_{j}}+\frac{\partial u_{j}^{\prime}}{\partial x_{i}}\right) \frac{\partial u_{j}^{\prime}}{\partial x_{i}}}}_{\epsilon}
\end{aligned}
$$

The terms are described in the following list:

- $C$ is the energy convection due to the particle movement, 
- $\Phi$ is the energy transport due to pressure effect,

$-T$ is the energy transport due to the vortices movement,

- $P$ is the energy production or the transfer of energy from the average movement to the turbulent movement,

- $D_{\mu}$ is the viscous diffusion that can be neglected in high Reynolds number flows [Blackman et al. (2017)],

$-\epsilon$ is the viscous dissipation.

In the present study, 2-components PIV measurements give access to the velocity components $(U, W)$. Under the assumption that the flow is $2 \mathrm{D}$ in the symmetry plane $\left(\bar{V}=0\right.$ and $\frac{\partial}{\partial y} \overline{()}=0$, for scales greater than the dissipation scale) and that the turbulence isotropic at dissipation scale, the different terms of the turbulent kinetic budget can be written as follows [Panigrahi et al. (2008), Gabriele et al. (2009)]:

$$
\begin{gathered}
k=\frac{3}{4}\left(\overline{u^{\prime} u^{\prime}}+\overline{w^{\prime} w^{\prime}}\right) \\
C=-\left(\bar{U} \frac{\partial k}{\partial x}+\bar{W} \frac{\partial k}{\partial z}\right) \\
T=-\left(\frac{1}{2} \frac{\partial}{\partial z} \overline{u^{\prime 2} w^{\prime}}+\frac{\partial}{\partial z} \overline{w^{\prime 3}}+\frac{1}{2} \frac{\partial}{\partial x} \overline{u^{\prime 3}}+\frac{\partial}{\partial x} \overline{u^{\prime} w^{\prime 2}}\right)
\end{gathered}
$$

under the assumption that $\overline{v^{\prime 2} w^{\prime}}=\overline{w^{\prime 3}}$ and $\overline{v^{\prime 2} u^{\prime}}=\overline{u^{\prime 3}}$.

$$
\begin{gathered}
P=-\left(\overline{u^{\prime} u^{\prime}} \frac{\partial \bar{U}}{\partial x}+\overline{w^{\prime} w^{\prime}} \frac{\partial \bar{W}}{\partial z}+\overline{u^{\prime} w^{\prime}} \frac{\partial \bar{U}}{\partial z}+\overline{u^{\prime} w^{\prime}} \frac{\partial \bar{W}}{\partial x}\right) \\
\epsilon=-15 \nu \overline{\left(\frac{\partial u^{\prime}}{\partial x}\right)^{2}}
\end{gathered}
$$

The local homogeneity has been checked for the first and second order statistics and it is assumed that the local isotropy is satisfied as it was done in similar studies [Panigrahi (2009)]. In the rest of the study, partial derivatives will be calculated using a second order centered finite difference method. $D_{\mu}$ is neglected and $\Phi$ is calculated as the rest of equation 2, assuming a steady flow state $\frac{\partial k}{\partial t}=0$. Figure 4 shows that the transverse component $\mathrm{V}$ is relatively unaffected by the cylinder wake. The velocity fluctuations at $z^{*}=2$ indicate a large shear stress area for $u^{\prime}$ and $w^{\prime}$ that does affect $v^{\prime}$. At $z^{*}=2$ for instance, values are $U / U \infty=0.64 \pm 0.2$, $V / V \infty=0.03 \pm 0.04$ and $W / W \infty=-0.05 \pm 0.16$. 
As stated in the introduction part, dissipation $\epsilon$ is often an issue in calculations. According to the theory [Kolmogorov (1941); Pope (2000)], dissipation occurs at scale smaller than the Kolmogorov scale $\eta$. Hence, the PIV resolution must be sufficient for direct $\epsilon$ calculations. In our case, PIV measurements can only resolve scales up to the size of the interrogation window: $11.6 \mathrm{~mm}$. Hence the PIV resolution is not sufficient to compute the dissipation with the direct method. That issue is not applicable for other components of the turbulent kinetic energy budget as they mainly occur at larger scales. Various calculation methods for $\epsilon$ exist [Sheng et al. (2000)] among which LE-PIV and ET methods. These methods applied to 2D PIV database propose an estimation of the dissipation from resolved Sub-Grid Scale (SGS). Both LE-PIV and ET methods are based on Large Eddy Simulation formalism. The dissipation is then evaluated using the following equations:

$$
\epsilon \sim \epsilon_{S G S}=-\overline{\tau_{i j} \widetilde{S}_{i j}}
$$

where $\tau_{i j}$ is the SGS stress tensor and $\widetilde{S}_{i j}$ is the filtered stress tensor

$$
\widetilde{S}_{i j}=\frac{1}{2}\left(\frac{\partial \widetilde{U}_{j}}{\partial x_{i}}+\frac{\partial \widetilde{U}_{i}}{\partial x_{j}}\right)
$$

The differences between both methods remain the evaluation of the stress tensor. Another class of method is the spectral method applied from temporal resolved database under several assumptions. After a brief recall of these three methods, they will be successively implemented to compute the TKE dissipation rate.

Spectral method.

The spectral method is based on the Richardson-Kolmogorov theory [Kolmogorov (1941)]. Under Homogeneous Isotropic Turbulence (HIT) conditions, at high Reynolds number, the theoretical one-dimensional energy spectrum in the inertial range is:

$$
E(f)=C_{k}^{\prime} \epsilon^{2 / 3} f^{-5 / 3} \text { with } C_{k}^{\prime}=C_{k}\left(\frac{\bar{U}}{2 \pi}\right)^{5 / 3}
$$

with the Kolmogorov constant $C_{k}$ equal to 1.5 [Pope (2000)]. Even if the constant value remains questionable [Al-Homoud and Hondzo (2007)].

Defining the constant $C_{k, 0}$ satisfying

$$
E(f)=C_{k 0} f^{-5 / 3}
$$


and corresponding to the intersection with the zero-axis of the slope in the log-log representation of the spectrum in the inertial range, the energy dissipation rate becomes:

$$
\epsilon=\left(\frac{C_{k 0}}{C_{k}}\right)^{3 / 2}\left(\frac{2 \pi}{\bar{U}}\right)^{5 / 2}
$$

The Kolmogorov scale can then be evaluated as follows:

$$
\eta=\left(\frac{\nu^{3}}{\epsilon}\right)^{1 / 4}
$$

Following previous developments, LDV measurements are used to compute the velocity fluctuations spectra. Figure 5 displays two representative spectra of the $u^{\prime}$ component which are superimposed on the $f^{-5 / 3}$ slope in the inertial range. Such a representation allows the evaluation of the Kolmogorov scale. Note that as a function of the spatial location, the spectral slope exhibits various $C_{k 0}$ values inducing different Kolmogorov scale values. The maximum value of $\eta$, is equal to $\eta=0.065 \mathrm{~mm}$ which is close to the $0.092 \mathrm{~mm}$ found in a rib wake in [Panigrahi et al. (2008)].
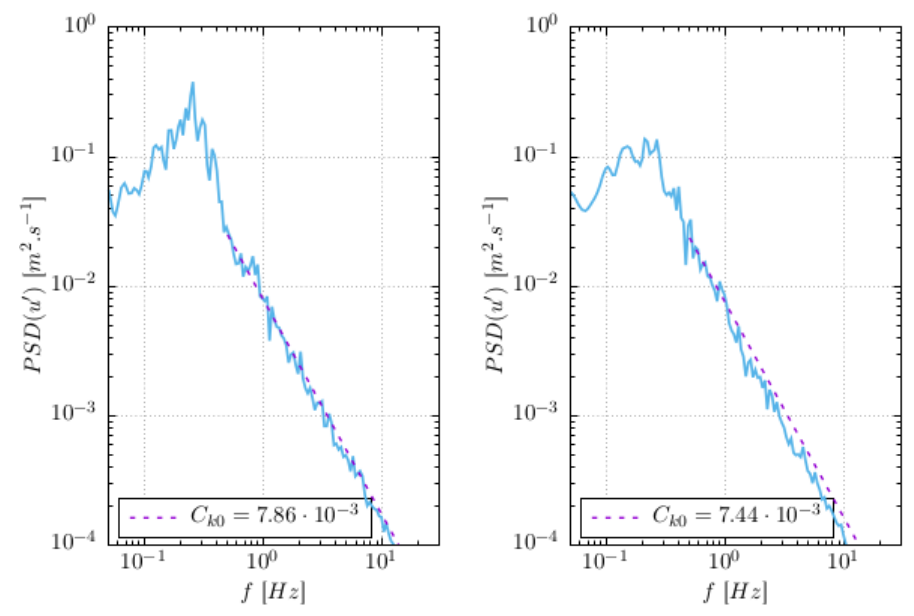

Figure 5: Spectra of the fluctuating streamwise velocity component superimposed on the slope approximation (equation 11, (dash red line) computed at two representative positions: Left: $x^{*}=9, z^{*}=2.2$. Right: $x^{*}=9, z^{*}=3.6$.

Direct Energy Transfer (ET) and LE-PIV methods.

Both ET and LE-PIV methods rely on the LES (Large Eddy simulation) formalism allowing a spatial scale filtering separation within the inertial range 
of the velocity spectrum. Details of the applications of these methods can be found in Sheng et al. (2000); Natrajan and Christensen (2006); Blackman et al. (2017). Consequently, the direct ET method uses the SGS stress tensor defined as follows:

$$
\tau_{i j_{E T}}=\widetilde{u_{i} u_{j}}-\widetilde{u_{i}} \widetilde{u_{j}}
$$

Based on the available 2D PIV database, an estimate of the SGS dissipation is given by Natrajan and Christensen (2006):

$$
\epsilon_{S G S}^{2 D}=-\left(\overline{\tau_{11} \widetilde{S}_{11}}+2 \overline{\tau_{12} \widetilde{S}_{12}}+\overline{\tau_{22} \widetilde{S}_{22}}\right)
$$

In the other hand, the LE-PIV method proposes to model the stress tensor using [Smagorinsky (1963)]:

$$
\tau_{i j}=-C_{s}^{2} \Delta^{2}|\widetilde{S}| \widetilde{S_{i j}}
$$

with $C_{s}=0.17$ the Smagorinsky constant and $\Delta$ the sub-grid size, which corresponds to the size of the spatial filtering. For isotropic turbulence and using PIV results, the dissipation rate can be written as [Khan (2005)]:

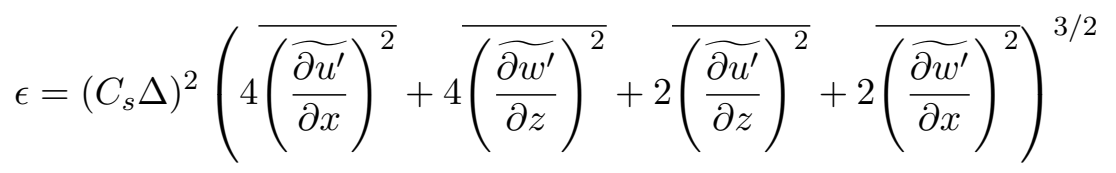

Both methods are implemented and successively tested using several sizes for the low-pass spatial filtering operation detailed below.

\subsection{TKE budget in the cylinder wake}

The upstream flow is anisotropic, but the turbulent agitation in the obstacles wake restores the isotropy [Ikhennicheu et al. (2017)]. In the symmetry plane, the cylinder wake can be considered 2D [Ikhennicheu et al. (2019c)]. The method described in the previous section to calculate the terms of the equation can thus be applied to the cylinder wake. Positive values are energy gains and negative values are energy losses. In the following the dissipation rate is estimated using the LE-PIV method.

Before presenting each term of the TKE budget, a statistical convergence analysis of the third order moments as well as the convection and transport terms of the TKE budget is presented. Figure 6 displays the following third 

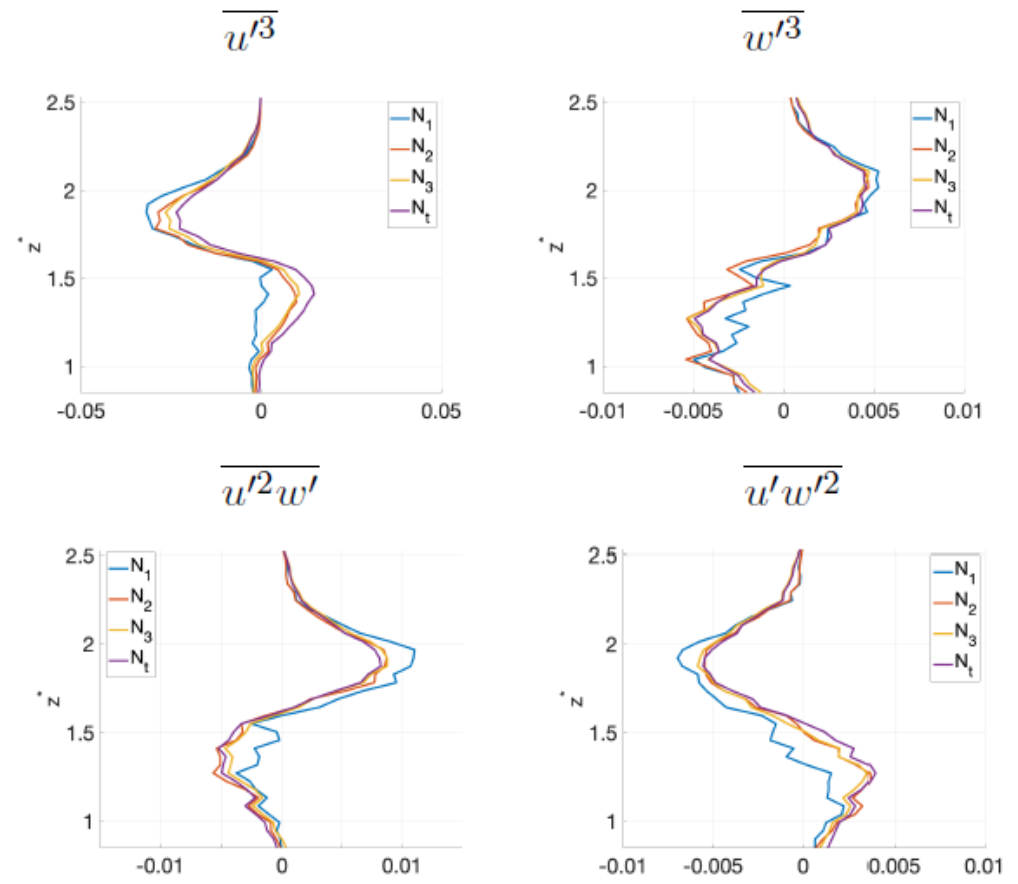

Figure 6: Illustration of the statistical convergence of the third-order moments along the $z$ direction $($ at $x=2) .\left(N_{1} ; N_{2} ; N_{3}\right)=(1000 ; 1500 ; 2000)$ and $N_{t}$ are successively considered to access the convergence of the moments.

order moments (used to compute the transport term of the TKE budget): $\overline{u^{\prime 3}}, \overline{w^{\prime 3}}, \overline{u^{\prime 2} w^{\prime}}, \overline{u^{\prime} w^{\prime 2}}$, calculated from $\left(N_{1} ; N_{2} ; N_{3} ; N_{t}\right)=(1000 ; 1500 ; 2000$; 2249) time steps.

It is observed that available $N_{t}$ time steps are sufficient to obtained quasi-converged third-order moment values. The statistical convergence is also done for the Convection $\mathrm{C}$ and Transport $\mathrm{T}$ terms (see Figure 7 ). Even if some very slight differences appear between $N_{3}$ and $N_{t}$, this representation confirms that the terms appearing in the TKE budget are statistically converged.

In order to choose and validate the value of $\Delta$, the size of the spatial filtering operation, we propose to determine the Sub Grid Scale (SGS) dissipation using varying values of $\Delta$. Both ET and LE-PIV methods are used to compute the dissipation by applying similar low pass filtering to velocity fields. Figure 8 displays the dissipation profiles computed from ET and LEPIV methods at $x^{*}=2$ using different values of $\Delta$. Note that the size of the spatial filtering is normalized with $d x$ the spatial resolution of PIV which 


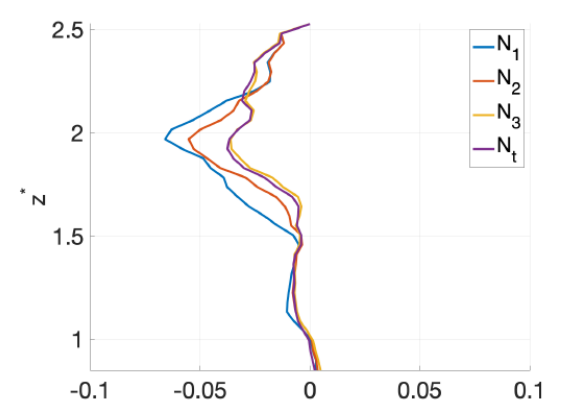

$T$

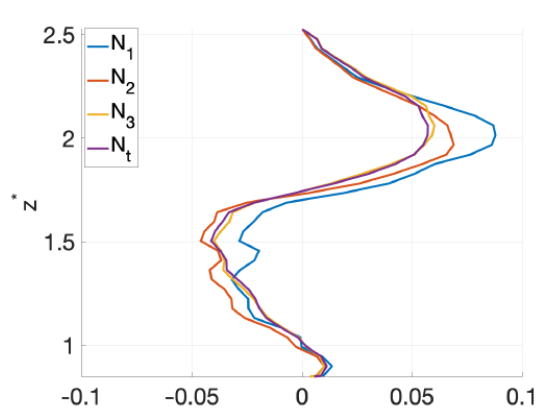

Figure 7: Illustration of the statistical convergence of the Convection (left had side) and Transport (right hand side) terms of the TKE energy budget along the $\mathrm{z}$ direction (at $x=2)$, based on $\left(N_{1} ; N_{2} ; N_{3}\right)=(1000 ; 1500 ; 2000)$ time steps.

is the limit size of the resolved small scales structures. First, ET results do not seem to be satisfactory fully converged for $\Delta \sim 1$, justifying that the profile of ET method with $\Delta=d x\left(\Delta^{*}=1\right)$ is not represented. Second, a quite good agreement is observed for both methods with ET dissipation values converging to LE-PIV values for $\Delta^{*}=1.23$. Based on these investigations, it is proposed to keep $\Delta^{*}=1.23$ value as a good compromise for both methods.

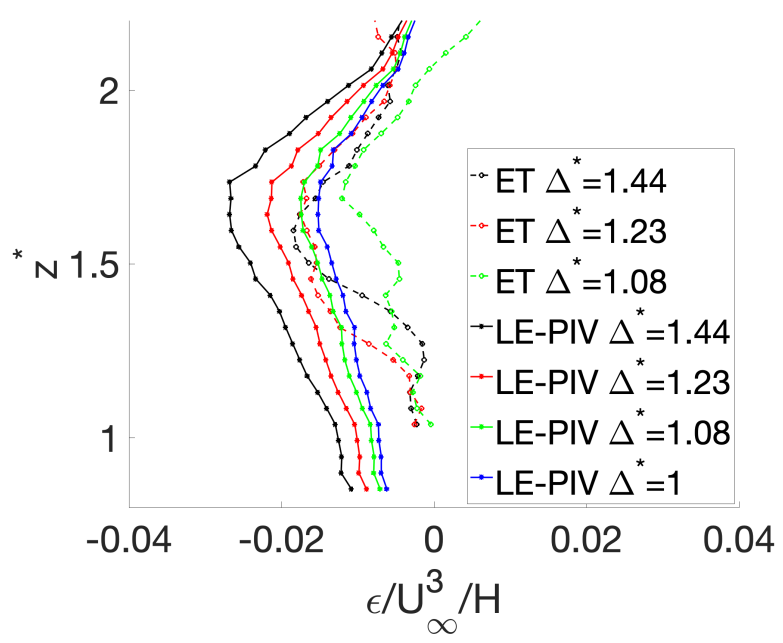

Figure 8: Comparison of dissipation terms calculated from ET and LE-PIV methods at $x^{*}=2$ using different values of $\Delta$, the size of the spatial filtering operation. $\left(\Delta^{*}=\Delta / d x\right)$ 
Figure 9 presents the distribution along the $z$ axis of each normalized term in the TKE budget equation. At $x^{*}=2$, the wake does not reach $z^{*}>4$, hence no measurements were carried out higher. Furthermore, figure 10 shows maps of production and dissipation in the very near wake.

Globally, for all quantities, their magnitude is relatively higher in the near wake compared to the far wake. The profiles also illustrate the shear layer, rising in the water column, with a decreasing intensity when moving further from the obstacle.

It is first confirmed that the main source of TKE is the vortex shedding developing behind the cylinder. A high level of the turbulent kinetic energy production $P$ is observed inside the shear layer area. The production peak occurs at the peak of the Reynolds shear stress. Indeed, Reynolds shear stress is the main contribution to turbulent energy production [Panigrahi et al. (2008); Panigrahi (2009); Blackman et al. (2017)]. Note that the high turbulent kinetic energy area (highlighted in figure 3) extends further downstream compared to that of turbulent production $P$ (presented in figure 8). Indeed, in the downstream direction $\left(x^{*}>5\right)$, the magnitude of the turbulent production gradually decreases due to the dissipation of the shear layer in the agitated turbulent flow. Figure 10 illustrates this decrease of the production intensity when the shear layer is dissipating. Production $P$ is compensated mostly by viscous dissipation $\epsilon$ at the peak, turbulent transport $T$ at the bottom and pressure transport $\Phi$ at the top.

Above the Reynolds shear stress peak, the dominant component is the turbulent transport $T$ from the shear layer to the outer flow. That effect is compensated by convection $C$ and pressure transport $\Phi$.

Convection $C$ is higher in the near-wake, due to the unsteady flow separation and presents generally small magnitude with negative values. The absolute value of $C$, which corresponds to the transfer of TKE via mean flow decreases in the far-wake as it was previously observed [Panigrahi (2009); Ricardo et al. (2014)].

Underneath the Reynolds shear stress peak, energy is mainly ejected by pressure effect. Hence, the turbulent kinetic energy produced by the shear is transported to the outer flow by turbulent processes $(T)$ and swept to the bottom by pressure gradient effects $(\Phi)$.

The turbulent transport term $T$ is then high in the shear layer and then changes sign inside the shear layer, negative in the lower region of the shear layer and positive in the upper region which is similar to previous studies [Panigrahi et al. (2008); Panigrahi (2009); Liu and Thomas (2004)].

Concerning the dissipation rate $\epsilon$, the TKE is dissipated within the shear 


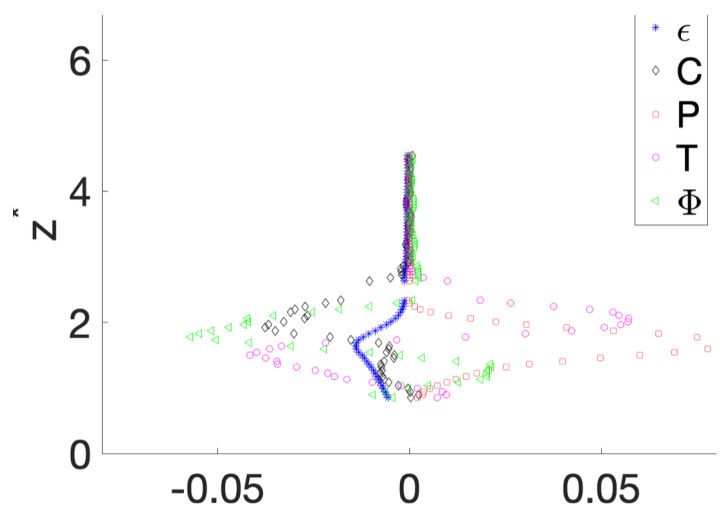

(a) $x^{*}=2$

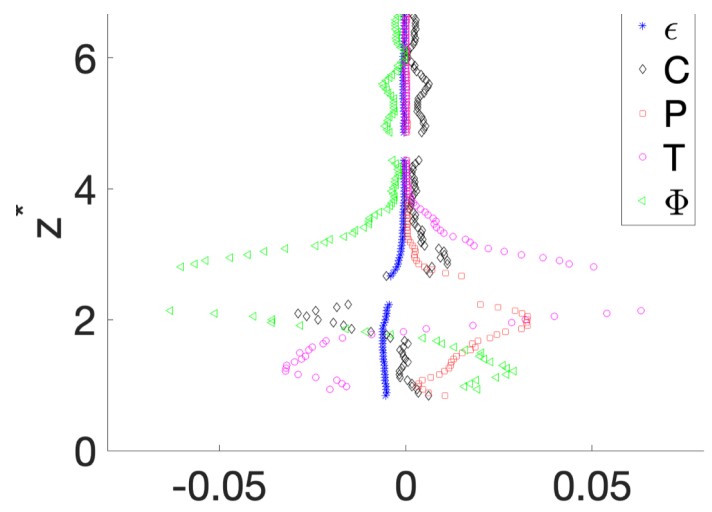

(b) $x^{*}=6$

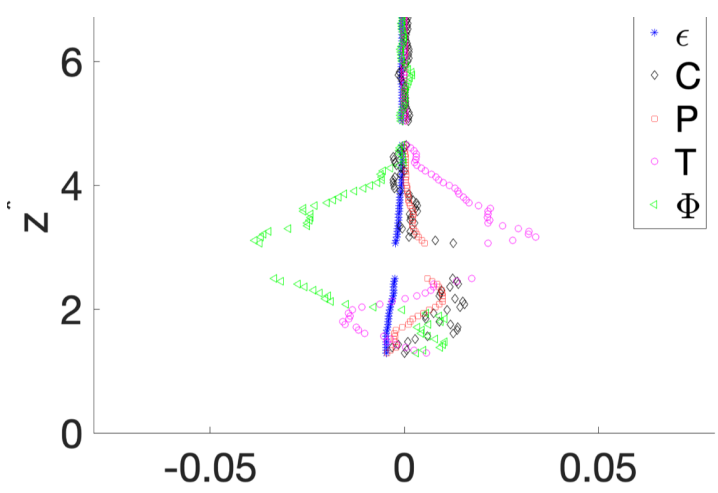

(c) $x^{*}=10$

Figure 9: Turbulent kinetic energy budget at three locations. 
layer especially in the near-wake. In this area, the dissipation rate follows the one of the turbulent production and its magnitude is maximal in the near wake (see figure 10). Dissipation $\epsilon$ is thus maximal at the position where turbulence intensity is the highest [Liu and Thomas (2004)] and is correlated to the production peak. That behaviour is often observed for flows past ribs [Panigrahi et al. (2008); Panigrahi (2009)], unlike for turbulent boundary layers. But in the far-wake, the dissipation rate does not seem to be directly related to the vortex shedding. To investigate the link between each term and the large scale flow structures, instantaneous and spectral analyses are performed in the next section.

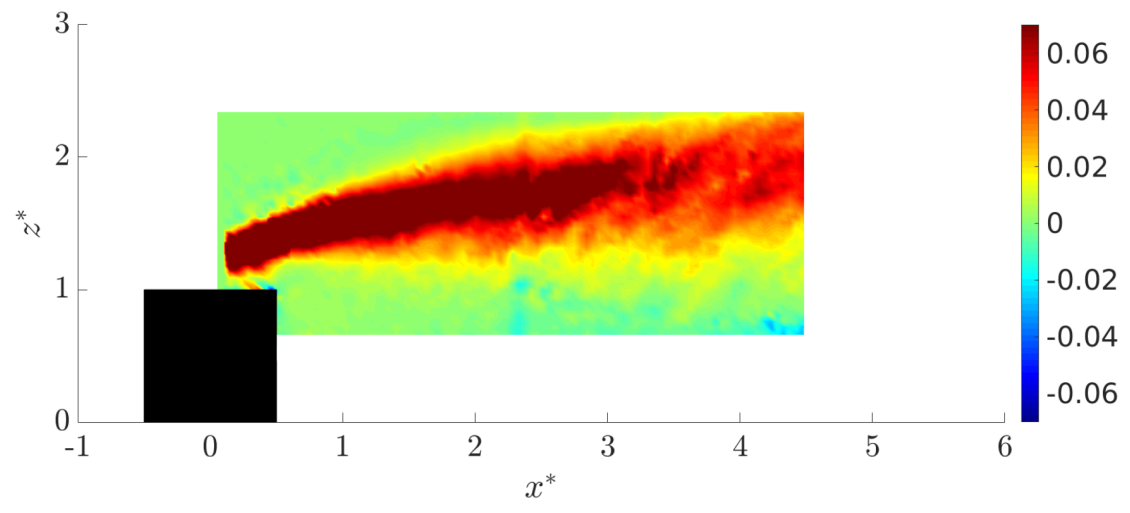

(a) Production $P /\left(U_{\infty}^{3} / H\right)$

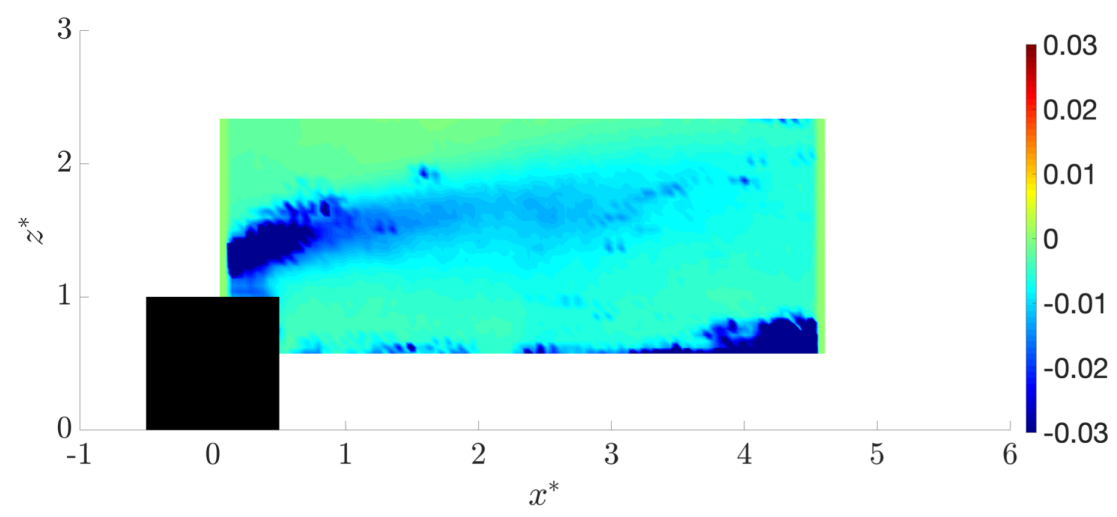

(b) Dissipation $\epsilon /\left(U_{\infty}^{3} / H\right)$

Figure 10: Maps of production $P$ and dissipation $\epsilon$ in the near wake 
Globally, along the symmetrical axis of the wall-mounted square cylinder in a high Reynolds flow, TKE budget terms exhibit the same tendency and amplitudes as the one presented in similar flow configurations for the flow past a wall-mounted rib at lower Reynolds numbers (see table 1) [Shah and Tachie (2008); Acharya et al. (1994); Panigrahi et al. (2008)]. A similar distribution of each TKE term is observed along the shear layer. Moving downstream, the intensities are lower and the production $P$ close to 0 due to the wake dissipation. The main components at those downstream positions are the turbulent transport $T$, and consequently the pressure transport $\Phi$, that can be caused by $3 \mathrm{D}$ effects.

Instantaneous and spectral analysis of production and dissipation terms. Instantaneous production is represented along with the fluctuating velocity signals in figure 11(a). It shows that production peaks occur when the fluctuating velocity drops, when a vortex is passing. Indeed, vortices shed in the cylinder wake present very intense negative fluctuating velocities in their bottom part and low positive velocities at the top [Ikhennicheu et al. (2019c)]. Hence, when a large scale turbulent structures passes, turbulent kinetic energy is produced. The production peak amplitude does not seem to be correlated to the velocity fluctuation intensity. Authors assume that it is due to the complex 3D organisation of vortex structures, a single point analysis is not enough to correlate vortices intensity to production peaks amplitude. Dissipation $\epsilon$, however, does not follow the velocity fluctuations. Indeed, the flow is dominated by large scale turbulent structures shed from the cylinder whereas dissipation occurs at small scales. Production and dissipation power spectral densities are also given in figure 11(b). The production spectrum follows a $-5 / 3$ slope, as for the velocity, in the inertial sub-range ([Kolmogorov (1941)]). There is a wide peak at $f \sim 0.3 \mathrm{~Hz}$, also present on the velocity spectra, indicating the vortex shedding frequency from the cylinder [Ikhennicheu et al. (2019c)]. Indeed, the vortices being shed are large scale structures, source of the production. Dissipation spectra does not follow the $-5 / 3$ slope and does not show any peak. Dissipation is thus not directly connected to the vortex shedding dominating the flow. Convection $C$ and turbulent transport $T$ signals and spectra are not depicted here, results are similar to the dissipation: their behaviour is not connected to the vortex shedding.

Investigation of the spectral method for dissipation rate estimation.

By comparing both methods (LE-PIV and spectral, using LDV data) for 

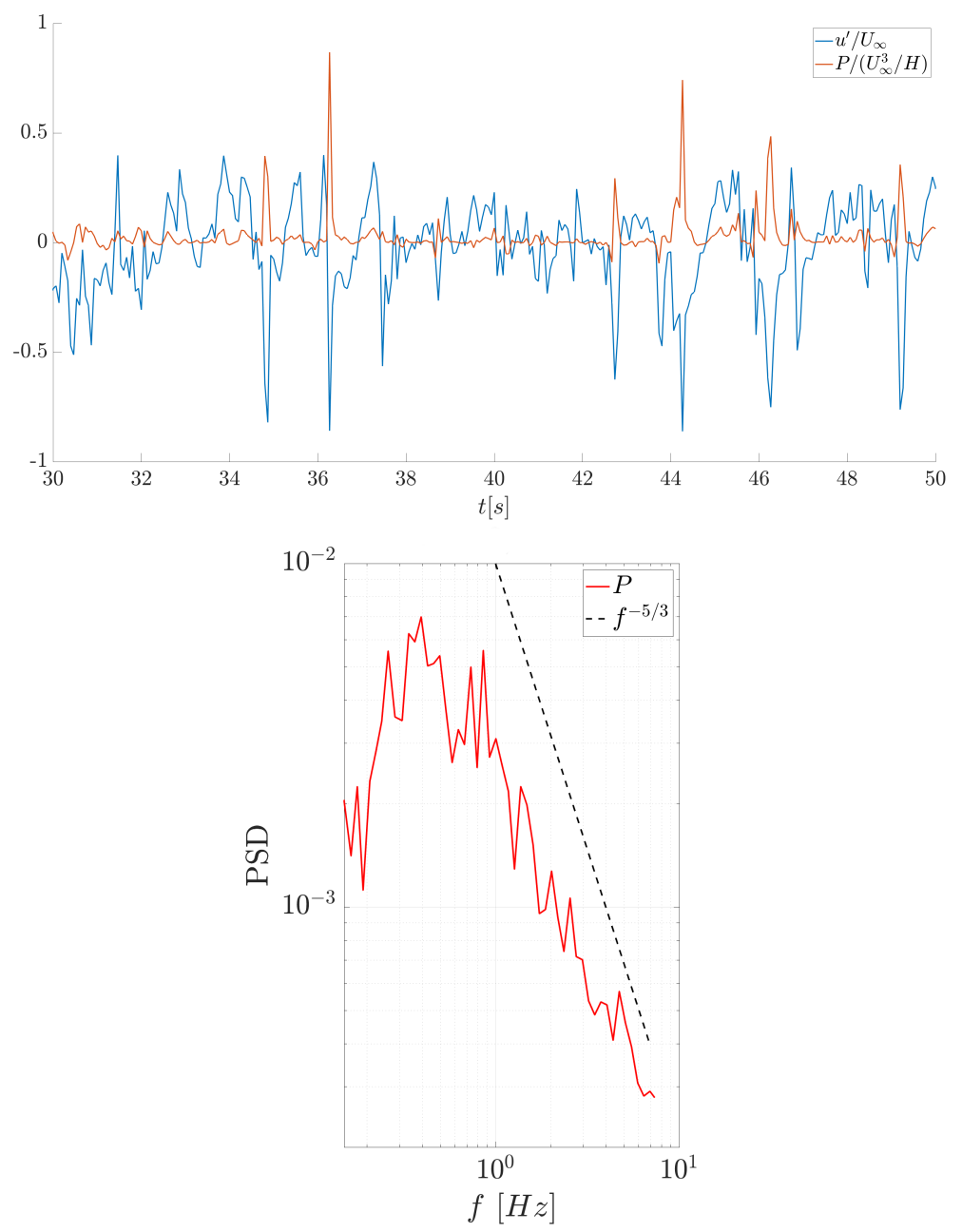

Figure 11: Signal of production $P$, dissipation $\epsilon$ and fluctuating velocity $u^{\prime}$ and PSD of $P$ and $\epsilon$ in $x^{*}=2, z^{*}=2$.

estimating the dissipation rate, it is possible to investigate the constant $C_{k}$ appearing in the spectral method and based on certains assumptions. Thus, from these results, we can also determine the Kolmogorov constant $C_{k}$ generally fixed at 1.5 [Tennekes and Lumley (1972)]. Indeed, numerous studies are questioning that value [Al-Homoud and Hondzo (2007)], looking to evaluate the dissipation in a undulating grid set-up, estimate that $0.43<C_{k}<4$. Differences observed between LE-PIV and the spectral method (figure 12) can thus be explained by a $C_{k}$ value to be adjusted using experimental data. 
In $x^{*}=2$, results from the spectral method are close to LE-PIV for $C_{k}=1$. Although LE-PIV can be used to question the $C_{k}$ values commonly used, as this method is based on strong assumptions, authors suggest that this value should be used in further works with precautions.

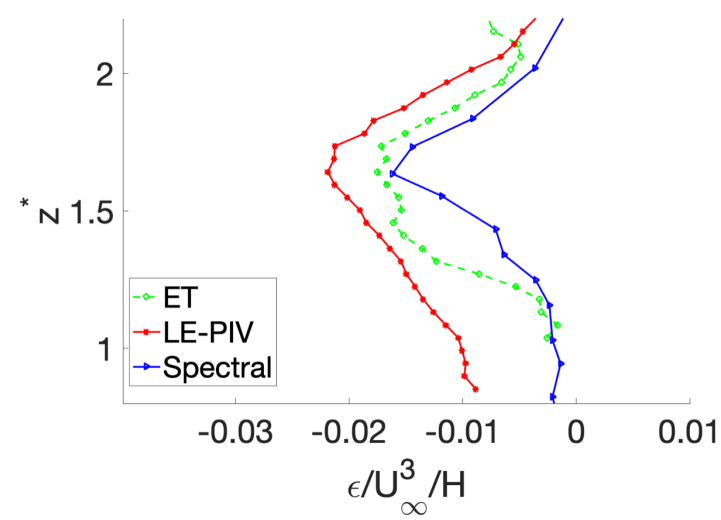

Figure 12: Comparison of dissipation term calculated from the three methods (direct Energy Transfer method, LE-PIV method, Spectral method) at $x^{*}=2$.

\section{Conclusions and Perspectives}

In this paper, specific in situ conditions are reproduced in the IFREMER flume tank at high Reynolds number $\left(R_{e}=2.5 \times 10^{5}\right)$. Large bathymetry variations are reproduced with a large aspect ratio wall-mounted cylinder. This obstacle produces a very energetic and extended wake embedded with large scale turbulent structures. Such turbulent inflow can have a strong impact on tidal turbines. The wake past the wall-mounted cylinder is thus investigated using PIV and LDV measurements. This paper focuses on the turbulent content of the cylinder wake using the decomposition of the turbulent kinetic energy budget equation. Different terms are identified and calculated using large scale PIV measurements planes (dimensions are $1153 \times 430 \mathrm{~mm}^{2}$ ). A special care is taken for the dissipation calculation, as viscous dissipation occurs at small scale, unlike for other terms. Using LDV measurements, the spectral method, based on the Richardson-Kolmogorov theory is used to determine the Kolmogorov scale at which viscous dissipation occurs. That scale is much smaller than the PIV interrogation window size. Hence, PIV measurements are not resolved enough to evaluate directly 
viscous dissipation. Consequently, the Large Eddy PIV is used, based on the Smagorinsky sub-grid model.

The kinetic energy budget is performed at different streamwise positions in the cylinder wake. Results show that production is maximized where the average Reynlods shear stress is the more intense, where energy transmitted from the mean flow to the turbulent flow is maximized. Dissipation peak occurs at the same position. In-between, turbulent kinetic energy is ejected to the outer flow by turbulent effects and swept to the bottom by pressure gradient effects. Further analysis shows that production peaks coincide with the passing of large scale turbulent structures. Dissipation, that occurs at smaller scales does not follow that trend.

That decomposition allows a more thorough analysis of the turbulent wake of the cylinder. However, dissipation computed with the LE-PIV is dependant on the subgrid scale and corresponds to the transfer of the TKE from resolved scales to subgrid ones. TKE budget decomposition may be used for validation of numerical model, allowing a more precise identification of any development issues. It also illustrates that the evaluation of the turbulent kinetic energy budget is achievable for large scale PIV planes in high Reynolds number flow. That type of description is useful when predicting the potential loads on a tidal turbine submitted to the wake of bathymetry elements. Furthermore, turbulent inflow conditions play a crucial role in the wake evolution behind the turbine. Then, the present thorough analysis of the energy exchanges is needed for the development of tidal turbine farm and specifically for the associated numerical models.

This paper presents the first results obtained for that study. Further experiments, including synchronous measurements of the three velocity components or PIV measurements planes of different size, are necessary to confirm the hypothesis for calculating the different components of the equation, even if the Large-Eddy PIV approach enabled to obtain full spatial maps of dissipation and also to compare it with the spectral method and to assess the validity of the constant used for the dissipation rate estimation.

The TKE energy budget can also be achieved at the turbine position, with or without its presence, in order to understand the complex interactions between the turbulent flow and the turbine behaviour. 


\section{Acknowledgment}

This work benefits from a French state grant managed by the National Research Agency under the Investments for the Future program bearing the reference ANR-10-IEED-0006-11. This work also received the support of the Met-Certified project, funding from the Interreg 2 Seas programme 2014-2020, co-funded by the European Regional Development Fund under subsidy contract N. 2S01-020. This project was partly financially supported by the European Union (FEDER), the French government, IFREMER and the region Hauts-de-France in the framework of the project CPER 2015-2020 MARCO. The authors also acknowledge the financial support of IFREMER and the Hauts de France Regional Council for this $\mathrm{PhD}$ study. We are most grateful to Thomas Bacchetti, Inès Belarbi, Jean-Valery Facq and the reviewers for their assistance and precious advices.

\section{References}

Acharya, S., Dutta, S., Myrum, T., and Baker, R. (1994). Turbulent flow past a surface-mounted two-dimensional rib. Journal of Fluids Engineering, 116:238-246.

Adrian, R. and Westerweel, J. (2011). Particle image velocimetry. Cambridge University Press, Cambridge.

Al-Homoud, A. and Hondzo, M. (2007). Energy dissipation estimates in oscillating grid setup: LDV and PIV measurements. Environmental Fluid Mechanics, 7:143-158.

Blackman, K., Perret, L., Calmet, I., and Rivet, C. (2017). Turbulent kinetic energy budget in the boundary layer developing over an urban-like rough wall using PIV. Physics of Fluids, 29:085113.

Castro, I. P., Cheng, H., and Reynolds, R. (2006). Turbulence over urbantype roughness: Deductions from wind-tunnel measurements. Boundary layer Meteo., 118:109-131.

Dey, S., Sarkar, S., Bose, S., Tait, S., and Castro-Orgaz, O. (2011). Wallwake flows downstream of a sphere placed on a plane rough wall. $J$. Hydraulic Eng., 137(10):1173-1189.

Durán Medina, O., Schmitt, F., Calif, R., Germain, G., and Gaurier, B. (2015). Correlation between synchronised power and flow measurements, 
a way to characterize turbulent effects on a marine current turbine. Proceedings of the 11th European Wave and Tidal Energy Conference, Nantes, France.

EDF (2010). Énergies marines hydrolienne et houlomotrice. Conférence institut Coriolis.

Gabriele, A., Nienow, A., and Simmons, M. (2009). Use of angle resolved PIV to estimate local specific energy dissipation rates for up- and downpumping pitched blade agitators in a stirred tank. Chemical Engineering Science, 64:126-143.

Hinze, J. (1975). Turbulence. 2nd Edition, McGraw-Hill, New York.

Ikhennicheu, M. (2019). Étude expérimentale de la turbulence dans les zones $\grave{a}$ forts courants et de son impact sur les hydroliennes. $\mathrm{PhD}$ thesis, Université de Lille.

Ikhennicheu, M., Druault, P., Gaurier, B., and Germain, G. (2017). An experimental study of influence of bathymetry on turbulence at a tidal stream site. Proceedings of the 12th European Wave and Tidal Energy Conference, Cork, Ireland.

Ikhennicheu, M., Gaurier, B., Germain, G., Druault, P., Pinon, G., and Facq, J.-V. (2019a). Experimental study of the wall-mounted cylinder wake effects on a tidal turbine behaviour compared to free stream turbulence. Proceedings of the 13th European Wave and Tidal Energy Conference, Napoli, Italy.

Ikhennicheu, M., Germain, G., Druault, P., and Gaurier, B. (2019b). Experimental investigation of the turbulent wake past real seabed elements for velocity variations characterization in the water column. International Journal of Heat and Fluid Flow, 78:108426.

Ikhennicheu, M., Germain, G., Druault, P., and Gaurier, B. (2019c). Experimental study of coherent flow structures past a wall-mounted square cylinder. Ocean Engineering, 182:137-146.

Khan, F. (2005). Investigation of turbulent flows and instabilities in a stirred vessel using particle image velocimetry. PhD thesis, Loughborough University. 
Kolmogorov, A. (1941). The local structure of turbulence in incompressible viscous fluid for very large Reynolds number. Doklady Akademii Nauk, 30:9-13.

Liu, X. and Thomas, F. (2004). Measurement of the turbulent kinetic energy budget of a planar wake flow in pressure gradients. Experiments in Fluids, 37:469-482.

Meinhart, C., Prasad, A., and Adrian, R. (1993). A parallel digital processor system for particle image velocimetry. Measurement Science Technology, 4:619-626.

Mercier, P. (2019). Modélisation de la turbulence engendrée par la morphologie du fond marin dans le Raz-Blanchard: Approche locale LBM-LES. $\mathrm{PhD}$ thesis, Université de Caen Normandie.

Mercier, P., Ikhennicheu, M., Guillou, S., Germain, G., Poizot, E., Grondeau, M., Thiébot, J., and Druault, P. (2020). The merging of kelvinhelmholtz vortices into large coherent flow structures in a high Reynolds number flow past a wall-mounted square cylinder. Ocean Engineering, 204:00-00.

Myers, L. and Bahaj, A. (2005). Simulated electrical power potential harnessed by marine current turbine arrays in the Alderney Race. Renewable Energy, 30:1713-1731.

Natrajan, V. K. and Christensen, K. T. (2006). The role of coherent structures in subgridscale energy transfer within the log layer of wall turbulence. Physics of Fluids, 18.

Ouro, P. and Stoesser, T. (2018). Impact of environmental turbulence on the performance and loadings of a tidal stream turbine. Flow, Turbulence and Combustion, 102.

Panigrahi, P. (2009). Piv investigation of flow behind surface mounted detached square cylinder. Journal of Fluids Engineering, 131(011202):1-16.

Panigrahi, P., Schroeder, A., and Kompenhans, J. (2008). Turbulent structures and budgets behind permeable ribs. Experimental Thermal and Fluid Science, 32:1011-1033.

Pope, S. (2000). Turbulent flows. Cambridge University Press, Cambridge. 
Ricardo, A., Koll, K., Franca, M., Schleiss, A., and Ferreira, R. (2014). The terms of turbulent kinetic energy budget within random arrays of emergent cylinders. Water resources research, 50:4131-4148.

Saarenrinne, P. and Piirto, M. (2000). Turbulent kinetic energy dissipation rate estimate from piv velocity vector fields. Experiments in Fluids, 29:300-307.

Shah, M. and Tachie, M. (2008). Flow relaxation past a transverse square rib in pressure gradients. AIAA Journal, 46(7).

Sheng, J., Meng, H., and Fox, R. (2000). A large eddy PIV method for turbulence dissipation rate estimation. Chemical Engineering Science, $55: 4423-4434$.

Smagorinsky, J. (1963). General circulation experiments with the primitive equations. Monthly Weather Review, 91:99-164.

Tennekes, H. and Lumley, J. (1972). A first course in turbulence. MIT press.

Westerweel, J. and Scarano, F. (2005). Universal outlier detection for PIV data. Experiments in Fluids, 39:1096-1100.

Zaripov, D., Li, R., and Dushin, N. (2019). Dissipation rate estimation in the turbulent boundary layer using high-speed planar particle image velocimetry. Experiments in Fluids, 60:18. 